\title{
Detection-localization Algorithms in the Around-the-corner Radar Problem
}

\author{
Khac-Phuc-Hung Thai*, Olivier Rabaste*, Jonathan Bosse*, Dominique Poullin* \\ Israel Hinostroza ${ }^{\dagger}$, Thierry Letertre ${ }^{\dagger}$ and Thierry Chonavel $^{\ddagger}$ \\ *ONERA, The French Aerospace Lab, France \\ ${ }^{\dagger}$ SONDRA, CentraleSupelec, France \\ ${ }^{\ddagger}$ IMT Atlantique, France \\ Email: kphung.thai@onera.fr
}

\begin{abstract}
Detection and localization in urban environments is a very recent radar problem. In this article, we investigate the possibility of detecting and locating targets in NLOS areas with a single portable radar by exploiting multipath returns. We propose two algorithms which handle the information provided by multipath returns in different ways to detect and estimate the NLOS target position. We also present an original method to select the number of paths to take into account in the algorithms in order to maximize detection probabilities. Numerical results show good efficiency of the proposed algorithms for problems of both detection and localization. We show that applying these algorithms improves detection performance compared to a classic matched filter in a typical urban scenario. Experimental results on a real data set allow us to validate our multipath model in urban environments, and in particular to show that it is possible to retrieve the target location even with rough knowledge of the scene geometry.
\end{abstract}

Keywords

around-the-corner radar, detection and localization algorithm, NLOS, multipath exploitation, urban environment, urban radar

\section{INTRODUCTION}

The around-the-corner radar is an emerging research topic for urban radar applications. Unlike conventional radar applications where targets are in direct line of sight (LOS) of the radar, the presence of buildings surrounding the radar in urban environments creates shadow areas. Targets in these areas are not in direct line of sight (NLOS) of the radar. However these NLOS targets may anyway be reached by reflections and/or diffractions on surrounding surfaces. These multipaths could be a challenge in conventional detection and localization problems due to the multiplicity of the echoes generated. Fortunately, in our context they can be exploited by the radar to detect and locate targets in shadow areas thanks to available knowledge of the scene geometry. It may then become possible to look around wall corners using a simple portable radar. Such a radar can be called "around-the-corner" radar (ACR) [1]. 


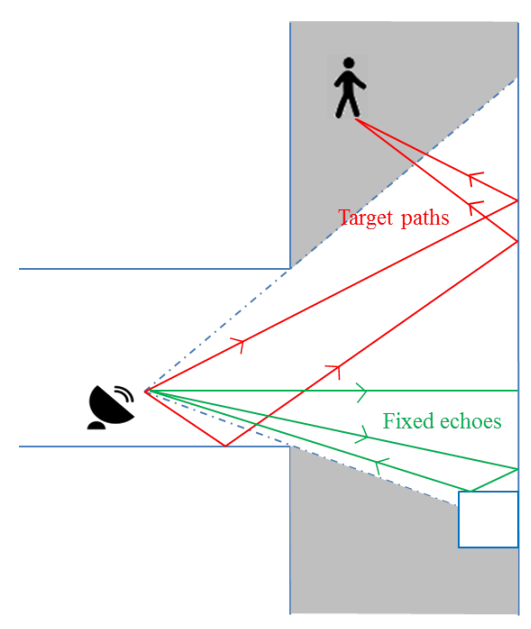

Fig. 1: Urban radar looking for targets in shadow areas

The objective of the ACR is thus to detect and locate NLOS targets by exploiting the multiple reflections induced by the urban environment. The topic is very recent in the literature. Preliminary experimental work was carried out to test the feasibility of detection in the absence of LOS with real measurements [1][2][3]. All these studies demonstrated that it is possible for a single radar to look around corners of walls by exploiting multipath information. Theoretical works aiming at exploiting multipath information are presented in [4] for airborne radar technologies, thus with a radar located far away and above the urban scene. Multipath was considered in [5] for a GMTI radar, and in [6] for a SAR technology with a ground-based radar.

In some other works [7][8][9][10], portable radars embedded in urban environment are used. In [8], the authors carried out several measurements in NLOS situations and focused on the micro-Doppler signature of the imaged target for the purpose of classifying objects. By extracting multipath information, the authors demonstrated that micro-Doppler features from walking persons can be retrieved. In [7], the authors proposed a multipath tracking algorithm and a location estimation based on a single reflection path, a single diffraction path and their combinations, by using geometrical calculation. Experimental results showed that the target was well located when information about at least two different paths was exploited. However the authors did not take into account two or more-bounce reflection paths on mirroring walls that appear in some scenes and could potentially improve the target localization.

In [9], detection of movements in a realistic scene was investigated. In this study, the authors applied an adaptive threshold level to the measured impulse response to detect moving targets. Results showed that detection of moving targets could be when one or two wall reflections are present between the transmitting antenna and the target. The method is relatively simple and easy to implement but information provided by different paths was processed separately. As a continuation of this work, in [10], a simulation was carried out for the problem of locating moving targets using a narrow beam antenna with $3 \mathrm{~dB}$ lobe width of $2.5^{\circ}$. Assuming that the geometry of the scene is known, the proposed localization solution was based mainly on detection distance found by applying an adapted threshold on the down-range profile, as described in [9], and knowledge of radar beam direction. With a narrow beam direction, the position of targets could be easily deduced from the detection distance and a simple ray tracing model. 
A simulation was carried out to demonstrate that targets can be detected and located with few false localizations. This solution assumed that the radar can provide an angular resolution small enough to cover all the supervised surface. However, this feature is not always available, which can cause localization errors.

Other recent work [11][12][13] exploited multipath with a single sensor for localization purposes only. In this work, the authors exploited multipath information by employing the time-of-arrival (TOA) estimated from the output of a classic matched filter. So they did not work directly with raw signals. The study [11] required that all paths are detected and should be labeled as direct or single-bounce paths and then associated to walls. In [13], this method was extended for passive radar to the double-bounce case, where, in addition, some paths may be undetected. Finally, the target location was found by intersecting of circles and ellipses characterized by the TOAs. Performance of these approaches was studied in [12]. Note that the problem of target detection was not considered in these articles. Target localization based on multipath exploitation was also investigated in tracking contexts [4][14].

In the present article, we consider the problem of detecting and locating a target in NLOS with a single portable radar. The transmitting antenna presents a wide beam ( $3 \mathrm{~dB}$ width equal to $\left.60^{\circ}\right)$. Thus the angle information for the paths is not known. Contrary to [11][13], we mainly focus on detection by exploiting the raw signal. Note that exploiting raw signals and not TOA measurements only is optimal from the estimation point of view [15]. Dealing with raw signals also makes it possible to avoid the potentially combinatorial association problem [15]. In addition, we do not assume that the number of multipath returns is constant: it depends on the scene geometry and configuration; in particular it may differ for two different target positions. Rough knowledge of the geometry of the scene is assumed. For instance, it could be extracted from open access street maps. A simple propagation model based on ray-tracing will be used to exploit the scene geometry. As such, the proposed solution can be applied to any geometrical configuration.

In this article, we develop two algorithms for target detection and localization. To the best of our knowledge, the problem of around-the-corner radar has not been treated with a dedicated multipath matched filter approach. Thus the first algorithm was developed in order to fill this gap by proposing a multipath propagation-based matched filter derived from a simple model. The algorithm jointly handles the information provided by multipath returns to detect and estimate the NLOS target position. It involves in a filter matched to the subspace spanned by the target. The scene geometry should be extracted from rough map information and only a rough propagation model can be used in the radar device for computational cost purposes. The proposed multipath subspace matched filter must therefore be robust to some misinformation about the exact scene geometry and wave propagation. In particular, no information regarding the amplitudes of the multiple paths is provided by the propagation model, assuming that such information cannot be obtained with only rough knowledge of the scene.

The second algorithm also works in the target state space directly. However, instead of using a matched filter, it involves in integrating the information from the different paths incoherently. As the ray-tracing model can produce a lot of paths for any single target position and the detection performance of the two algorithms depends on the number of paths taken into account, a selection algorithm for the optimal number of paths is also proposed.

Simulations will be carried out to evaluate the performance of these two algorithms with respect to two aspects: detection and localization. It will be shown that in a particular NLOS urban configuration, using the two algorithms can improve detection performance compared to the classic matched filter. For the purpose of localization, both algorithms provide promising results, despite some spatial ambiguities. 
A real radar experiment was also carried out with a small portable radar. It showed that a simple propagation model can explain most of the multipath return images provided by a portable radar in an NLOS case. The results obtained show that in real scenes, it is possible to retrieve the target location even with only rough knowledge of the scene geometry. Note that part of the experimental work was presented in [16].

The article is organized as follows. In section II, the signal model will be introduced. In section III, two detection and localization algorithms will be presented. In the next section, a selection algorithm will be proposed to find the optimal number of multiple paths for these two algorithms. In section V, results of the detection-localization algorithms and the selection algorithm will be presented for simulated data. Finally, in section VI, a real experiment will be introduced and experimental results of the detection-localization algorithms will be shown and analyzed.

\section{SignAL MODEL}

In the following, we assume that all reflections are specular, and that diffraction effects can be neglected. The Doppler information is naturally present in the signal and exploited to remove the fixed (zero Doppler) echoes. However, for the purpose of simplicity and limitation of the hardware configuration used in the experimentation, we will not exploit the Doppler information of the different paths for target detection-localization. Of course, exploitation of the Doppler would be of interest to improve the localization performance of the proposed solution. We also assume here that strong fixed echoes produced by the surrounding buildings and objects have already been removed. This can be done for instance by applying a zero-Doppler rejection method based on [17] or [18]. So, the only received echoes are backscattered by nearby moving targets. In the following, a single target is considered.

The problem of detecting and locating a single target in a 2D-surface problem is considered. An example of a typical considered scenario is provided in Fig. 1. It represents a simple urban setting, where the radar located in the main street is looking for targets located in the adjacent perpendicular street.

The signal $y(t)$ after cancellation of fixed echoes received by the radar in the urban environment can be written, taking into account the multipath propagation, as

$$
y(t)=\sum_{k=1}^{M(x, y)} \alpha_{k} s\left(t-\tau_{k}(x, y)\right)+n^{\prime}(t),
$$

where $s(t)$ is the signal transmitted by the radar, $M(x, y)$ is the number of multipath returns for a target located at position $(x, y), \tau_{k}(x, y)$ is the delay of the $k$-th return for this target and $\alpha_{k}$ is its amplitude. $n^{\prime}(t)$ is assumed to be a circular complex white Gaussian noise. In order to separate the different multipaths in range, it is important to use a signal with a sufficiently large bandwidth, typically several hundreds of MHz. This implies a high sampling frequency. So, once sampled, the received signal $y(t)$ can contain a lot of samples (typically thousands of samples), although all the information is contained in a few tenths of range cells. Applying a range matched filter enables to concentrate this information in a few range cells and get rid of unuseful remaining cells, thus saving calculation time and memory. Letting $z(t)$ denote the output of the matched filter applied to $y(t)$, we have:

$$
z(t)=\sum_{k=1}^{M(x, y)} \alpha_{k} r\left(t-\tau_{k}(x, y)\right)+n(t),
$$

where $r(t)$ is the autocorrelation of $s(t)$ and $n(t)$ is the output of the matched filter applied to $n^{\prime}(t)$. 
We take $N$ snapshots $t_{i}=i T_{s}=i / f_{s}(i=1, \ldots, N)$, where $f_{s}$ is the sample frequency. $N$ is taken large enough to measure the overall channel length for all locations, we define the following vectors and matrices:

$$
\begin{gathered}
\mathbf{r}(t-\tau)=\left[\begin{array}{llll}
r\left(t_{1}-\tau\right) & r\left(t_{2}-\tau\right) & \ldots & r\left(t_{N}-\tau\right)
\end{array}\right]^{T} \\
\mathbf{n}=\left[\begin{array}{llll}
n\left(t_{1}\right) & n\left(t_{2}\right) & \ldots & n\left(t_{N}\right)
\end{array}\right]^{T} \\
\mathbf{z}=\left[\begin{array}{llll}
z\left(t_{1}\right) & z\left(t_{2}\right) & \ldots & z\left(t_{N}\right)
\end{array}\right]^{T} \\
\boldsymbol{\alpha}=\left[\begin{array}{llll}
\alpha_{1} & \alpha_{2} & \ldots & \alpha_{M(x, y)}
\end{array}\right]^{T} \\
\mathbf{R}(x, y)=\left[\begin{array}{lll}
\mathbf{r}\left(t-\tau_{1}(x, y)\right) & \mathbf{r}\left(t-\tau_{2}(x, y)\right) & \ldots \\
\mathbf{r}\left(t-\tau_{M(x, y)}(x, y)\right)
\end{array}\right]
\end{gathered}
$$

The equation (1) can be then written as

$$
\mathbf{z}=\mathbf{R}(x, y) \boldsymbol{\alpha}+\mathbf{n}
$$

The sampling frequency is set at the signal bandwidth. So $\mathbf{n}$ is also a circular complex white Gaussian noise. The noise covariance matrix is assumed to be $\sigma^{2} \mathbf{I}$ with $\sigma^{2}$ known.

In order to apply this signal model to our particular problem, the area of interest for target detection is divided into small cells as seen in Fig. 2. A simulation was carried out with a rough geometrical model of a urban intersection: doors, windows and other possible backscattering elements were not modeled. Signal propagation is modeled by a simple ray tracing, assuming that all reflections are specular. Propagation losses are simulated using a simple model: loss is proportional to the square of the distance traveled by the wave from the radar to the target and back to the radar, according to the classical radar equation [19]. Reflection losses are also added by considering a $3 \mathrm{~dB}$ constant loss per reflection. For each cell $(x, y)$, the matrix $\mathbf{R}(x, y)$ with $M(x, y)$ columns is generated, where $M(x, y)$ is the number of multiple paths for this cell. Multipath delays $\tau_{k}(\mathrm{k}=1,2, \ldots, M(x, y))$ for a target located in a given cell $(x, y)$ are calculated from the ray tracing simulation. Although many multiple paths may be simulated with a large number of reflections, it is possible to consider only a reduced number of paths for the detection-localization algorithm, as paths presenting too many reflections will be too weak to provide exploitable information. Then, once the multipath delays are computed, the matrix $\mathbf{R}(x, y)$ corresponding to cell $(x, y)$ can be easily found.

\section{DETECTION AND LOCALIZATION ALGORITHMS}

In classic radar problems, the detection test involves applying a threshold to the matched filter output (possibly normalized), which corresponds to a generalized likelihood ratio test (GLRT) strategy. However, this type of strategy, which does not take into account the presence of multiple paths, but only the information of a single path, is generally insufficient to locate the target in an urban environment. The consideration of multiple paths should make it possible to extract more information about the contribution of an NLOS moving target to the observation. This reduces ambiguities in localization and improves detection performance. The following two algorithms are developed to take into account information from different paths simultaneously. 


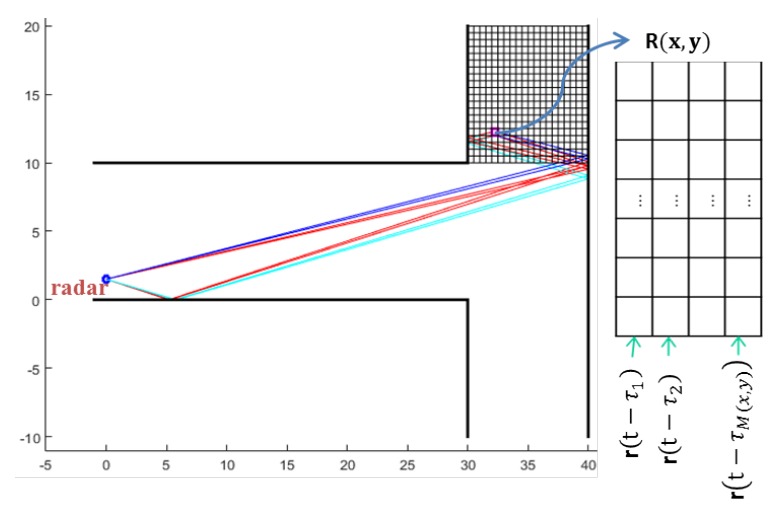

Fig. 2: Typical urban scenario

\section{A. Multipath matched filter (MPF)}

Following the Direct Position Determination (DPD) approach [15], we will work in the target space $(x, y)$ directly. This seems particularly relevant in our context where inferring location directly from path delays is difficult, due to multipath propagation.

For a fixed position $(x, y)$, the hypothesis testing problem to be considered is the following:

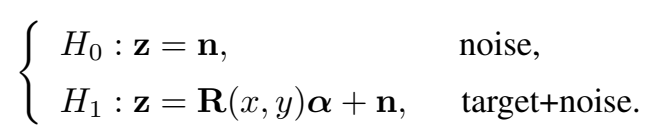

Exploiting prior information on the path amplitudes $\boldsymbol{\alpha}$ is not trivial, since this would require detailed knowledge of the wall materials and reflectivities, as well as the thorough knowledge of the target radar cross section for all angles. Instead, we assume here that these amplitudes are unknown and deterministic. The only knowledge we extract from the propagation model is thus the information about the path delays. Classically, in such a setting, undesired unknown parameters are estimated from the Maximum Likelihood (ML) criteria in order to eliminate them from the decision statistics. The ML estimation of the amplitude vector $\alpha$ is provided by

$$
\hat{\boldsymbol{\alpha}}=\left(\mathbf{R}^{H}(x, y) \mathbf{R}(x, y)\right)^{-1} \mathbf{R}^{H}(x, y) \mathbf{z} .
$$

Thus, injecting this expression into the likelihood ratio corresponding to the above hypothesis testing problem provides the following GLRT for our detection problem [20]:

$$
T_{M P F}(x, y) \underset{H_{0}}{\stackrel{H_{1}}{\gtrless}} \lambda_{M P F},
$$

where

$$
T_{M P F}(x, y)=\|\mathbf{P}(x, y) \mathbf{z}\|^{2}
$$

and

$$
\mathbf{P}(x, y)=\mathbf{R}(x, y)\left(\mathbf{R}^{H}(x, y) \mathbf{R}(x, y)\right)^{-1} \mathbf{R}^{H}(x, y)
$$


is the projection onto the subspace spanned by the columns of the matrix $\mathbf{R}(x, y)$.

For a fixed position $(x, y)$, this GLRT solution is known as the matched subspace detector [21]. It aims at detecting signals belonging to the subspace spanned by matrix $\mathbf{R}(x, y)$.

Under $H_{0}$ hypothesis, the probability distribution of $T_{M P F}^{\prime}(x, y)=T_{M P F}(x, y) / \sigma^{2}$ is a $\chi^{2}$ central distribution with $2 M(x, y)$ degrees of freedom. Thus the threshold level $\lambda$ can be calculated as a function of the number of multiple paths $M(x, y)$, the noise variance and the false alarm probability $P_{F A}$, as follows:

$$
\lambda_{M P F}=2 \sigma^{2} \gamma^{-1}\left(M(x, y), \Gamma(M(x, y))\left(1-P_{F A}\right)\right)
$$

where $\Gamma(N)$ and $\gamma(N, x)$ are the gamma and the lower incomplete gamma functions respectively.

The detection probability is provided by:

$$
P_{d(M P F)}=Q_{M(x, y)}\left(\frac{\|\mathbf{R}(x, y) \boldsymbol{\alpha}\|}{\sigma}, \frac{\sqrt{\lambda_{M P F}}}{\sigma}\right),
$$

where $Q_{M}(a, b)$ is the Marcum Q-function. It is defined as:

$$
Q_{M}(a, b)=\int_{b}^{\infty} x\left(\frac{x}{a}\right)^{M-1} \exp \left(-\frac{x^{2}+a^{2}}{2}\right) I_{M-1}(a x) d x
$$

where $I_{M}(z)$ is the modified Bessel function with order $M$ [22]. The derivation for (8) and (9) can be found in Appendix A.

For the problem of localization, still considering that $\alpha$ is an unknown deterministic vector, the ML estimator of position $(x, y)$ in (3) is given by the maximum of $T_{M P F}(x, y)$. Like in a classic LOS radar, the single target ML cost function can then be used both for detection and localization. The value $T_{M P F}(x, y)$ (called MPF T-level) for the detection test at each position $(x, y)$ in the considered zone is calculated. The cells with high T-level are more likely to correspond to the position of the target. In the monotarget setting, an estimation of the target position can be found as

$$
(\hat{x}, \hat{y})=\underset{x, y}{\operatorname{argmax}}\left(T_{M P F}(x, y)\right)
$$

In order to apply the algorithm to our particular problem, the zone considered for target detection is divided into small cells as seen in Fig. 2. For each cell, a matrix $\mathbf{R}(x, y)$ is computed from the ray-tracing model.

Let us note that the choice of $M(x, y)$ influences the detection performance as the threshold and the matrix $\mathbf{R}(x, y)$ change with $M(x, y)$. In section IV, we propose an original method to determine $M(x, y)$.

\section{B. Square law combiner (SLC)}

As described above, the multipath matched filter requires memory for stacking parameters obtained from the ray-tracing model and/or the calculation of a matrix inverse for each tested position. The calculation time and memory requirements can dramatically increase when the number of tested positions is large. So, we propose a second algorithm that enables to reduce both factors. Instead of estimating the amplitude vector $\boldsymbol{\alpha}$ for the matched filter, this algorithm computes an incoherent integration of the range matched filter output for the multipath delays provided by the propagation model. As such, it shares some similarities with the square law combiner technique considered in radio transmissions [23]. 
The hypothesis test of the SLC algorithm is :

$$
T_{S L C}(x, y)=\sum_{i \in \Omega(x, y)}|\mathrm{z}(\mathrm{i})|^{2} \underset{H_{0}}{\stackrel{H_{1}}{\gtrless}} \lambda_{S L C},
$$

where $\Omega(x, y)$ contains the index of the vector $\mathbf{z}$ corresponding to the multipath delays $\tau_{k}(x, y)$ obtained by the ray-tracing model. The number of elements in $\Omega(x, y)$ is then equal to $M(x, y)$.

It can be easily shown that the probability law of $T_{S L C}^{\prime}(x, y)=T_{S L C}(x, y) / \sigma^{2}$ under $H_{0}$ hypothesis is a $\chi^{2}$ central distribution with $2 M(x, y)$ degrees of freedom. Thus the threshold level $\lambda$ can be calculated exactly as for the previous algorithm:

$$
\lambda_{S L C}=2 \sigma^{2} \gamma^{-1}\left(M(x, y), \Gamma(M(x, y))\left(1-P_{F A}\right)\right)=\lambda_{M P F}
$$

The detection probability is in that case provided by:

$$
P_{d(S L C)}=Q_{M(x, y)}\left(\frac{\sqrt{\sum_{i \in \Omega(x, y)}\left|[\mathbf{R}(x, y) \boldsymbol{\alpha}]_{i}\right|^{2}}}{\sigma}, \frac{\sqrt{\lambda_{S L C}}}{\sigma}\right),
$$

where $[\mathbf{R}(x, y) \boldsymbol{\alpha}]_{i}$ is the $i$-th entry of the vector $\mathbf{R}(x, y) \boldsymbol{\alpha}$.

Like in the MPF algorithm, the value $T_{S L C}(x, y)$ (called SLC T-level) for the detection test in the considered zone can be calculated for each position $(x, y)$. The cells with high T-level are more likely to correspond to the position of the target and the target location is estimated as:

$$
(\hat{x}, \hat{y})=\underset{x, y}{\operatorname{argmax}}\left(T_{S L C}(x, y)\right)
$$

\section{Comparison of detection performance}

It appears that the expression of $P_{d(S L C)}$ only differs from the expression of $P_{d(M P F)}$ by the value of the noncentrality parameter. Thus, for a same $P_{F A}$ and a same number of paths $M(x, y)$, we have $P_{d(M P F)}>P_{d(S L C)}$ as $\|\mathbf{R}(x, y) \boldsymbol{\alpha}\|$ is always greater than $\sqrt{\sum_{i \in \Omega(x, y)}\left|[\mathbf{R}(x, y) \boldsymbol{\alpha}]_{i}\right|^{2}}$ and the Marcum Q-function increases with the first parameter [24]. Therefore, the MPF detection performance is always better than the SLC one.

\section{Comparison of computation time and memory requirement}

The MPF algorithm requires the computation of matrices $\mathbf{P}(x, y)$ for each location $(x, y)$. These matrices are provided by the propagation model. Running the propagation model for each location at each iteration may be very costly. However, the MPF algorithm can be implemented in different ways. First, in order to decrease the computation time, we can stack $N \times N$ matrices $\mathbf{P}(x, y)$ provided by Eq.(7) for all cells of the area of interest. In this way, we avoid matrix inverse computation so we decrease time cost but increase the memory requirement. Another implementation can be stacking $N \times M(x, y)$ matrices $\mathbf{R}(x, y)$ for all cells and computing $\mathbf{P}$ by Eq.(7) at each program launch, decreasing thus the memory requirement but increasing the computation time. The last way is to only stack parameters of matrices $\mathbf{R}$ (delays $\tau_{k}(x, y)$ and the autocorrelation signal $r(t)$, and constructing $\mathbf{R}$ and computing $\mathbf{P}$ at each program launch. An example of memory requirement and computation time comparison between the SLC algorithm and three implementations of the MPF algorithm is shown in the Table 1. 
It is shown that the SLC algorithm provides a big gain, both in time and memory requirement. Depending on the implementation, the MPF algorithm can be fast but greedy in memory, or vice versa.

\begin{tabular}{|c|c|c|}
\hline & Time (s) & Memory (MB) \\
\hline SLC & 0.02 & 0.04 \\
\hline MPF: stacking P & 0.20 & 2145 \\
\hline MPF: stacking R & 0.92 & 22 \\
\hline MPF: stacking parameters of R & 1.27 & 0.04 \\
\hline
\end{tabular}

TABLE I: Memory requirement and computation time comparison. $N=300, M(x, y)=3$, area of interest with 40x40 cells. Matlab environment, $2.8 \mathrm{GHz}$ PC.

\section{SElecting the NUmber OF MUltiple PATHS}

As noted in the previous section, the choice of the number of paths taken into account influences the detection performance of the MPF and SLC algorithms. In this section, we will first show that taking into account additional path returns can improve detection performance. Then, we will propose an original method to select the optimal number of paths, based on a detection performance criteria. The following detection algorithm is developed with the MPF algorithm because of its better performance compared to the SLC.

\section{A. Detection performance comparison : $N$ vs $N+1$ paths}

We compare the detection performance of the MPF with $N$ and $N+1$ paths respectively. We will show that taking into account an additional path in the detection process can increase the detection performance.

Without loss of generality, let $\|\mathbf{r}\|=1$ and $\sigma_{n}^{2}=1$ so that $\left|\alpha_{i}\right|$ represents the signal-to-noise ratio (SNR) of the $k$-th path respectively. For the sake of simplicity, we assume that $\left|\alpha_{1}\right| \geq\left|\alpha_{2}\right| \geq \ldots \geq\left|\alpha_{n}\right| \geq\left|\alpha_{n+1}\right|$ and we note $\boldsymbol{\alpha}_{1: n}=\left[\alpha_{1}, \alpha_{2}, \ldots, \alpha_{n}\right]$.

We will also assume that $\mathbf{r}\left(t-\tau_{i}\right)$ and $\mathbf{r}\left(t-\tau_{j}\right)$ are uncorrelated for any pair $(i, j)$, which is approximately the case when the two considered paths are not too close. Or, to be more specific, when $\left|\tau_{i}-\tau_{j}\right|$ is greater than $1 / B$. The detection probability of the MPF with $n$ multipaths is then

$$
P_{d}^{(n)}\left(\boldsymbol{\alpha}_{1: n}\right)=Q_{n}\left(\sqrt{2 \sum_{k=1}^{n}\left|\alpha_{k}\right|^{2}}, \sqrt{2 t_{n}}\right)
$$

where $t_{n}=\gamma^{-1}\left(n, \Gamma(n)\left(1-P_{f a}\right)\right)$.

Similarly, the detection probability of the MPF with $n+1$ multipath is

$$
P_{d}^{(n+1)}\left(\boldsymbol{\alpha}_{1: n+1}\right)=Q_{n+1}\left(\sqrt{2 \sum_{k=1}^{n+1}\left|\alpha_{k}\right|^{2}}, \sqrt{2 t_{n+1}}\right)
$$

where $t_{n+1}=\gamma^{-1}\left(n+1, \Gamma(n+1)\left(1-P_{f a}\right)\right)$.

Here, we would like to determine which solution provides the best $P_{d}$, and for which set of parameters. This results in comparing the two quantities $P_{d}^{(n)}\left(\boldsymbol{\alpha}_{1: n}\right)$ and $P_{d}^{(n+1)}\left(\boldsymbol{\alpha}_{1: n+1}\right)$ for different values of $\left|\alpha_{k}\right|$, that is, comparing 


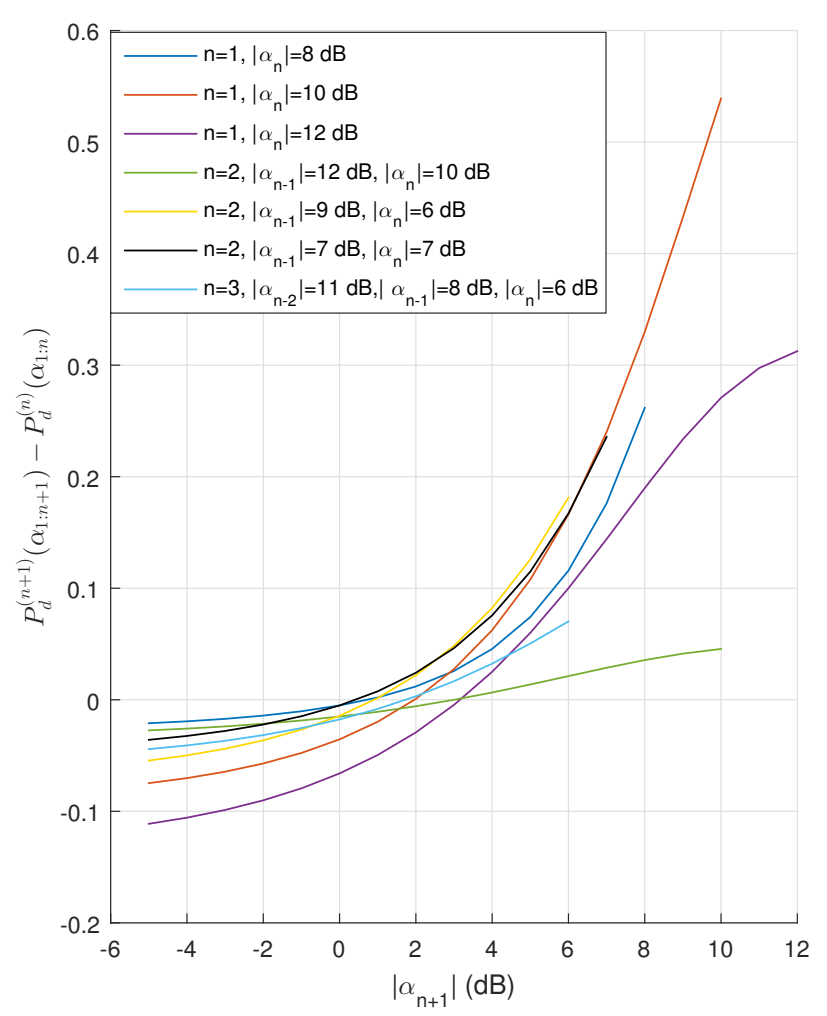

Fig. 3: $P_{d}^{(n+1)}\left(\boldsymbol{\alpha}_{1: n+1}\right)-P_{d}^{(n)}\left(\boldsymbol{\alpha}_{1: n}\right)$ for different values of $n$

two Marcum Q-functions with three different parameters $M, a$ and $b$. It is shown that $Q_{M}(a, b)$ increases with $M$ and $a$ but decreases with $b$ [24]. Therefore, it is not trivial to compare $P_{d}^{(n)}\left(\boldsymbol{\alpha}_{1: n}\right)$ and $P_{d}^{(n+1)}\left(\boldsymbol{\alpha}_{1: n+1}\right)$. To the best of our knowledge, this complex comparison has not been studied yet in the literature.

Fig. 3 shows some examples of the variation of $P_{d}^{(n+1)}\left(\boldsymbol{\alpha}_{1: n+1}\right)-P_{d}^{(n)}\left(\boldsymbol{\alpha}_{1: n}\right)$ as a function of $\left|\alpha_{n+1}\right|$ for some specified values of $n$ and $\left|\boldsymbol{\alpha}_{1: n}\right|$. We observe that there is always one and only one intersection between the curves $P_{d}^{(n+1)}\left(\boldsymbol{\alpha}_{1: n+1}\right)-P_{d}^{(n)}\left(\boldsymbol{\alpha}_{1: n}\right)$ and the zero horizontal axis. From many other numerical simulations obtained with other values of $\left|\boldsymbol{\alpha}_{1: n}\right|$ and larger values of $n$, we conclude that seems to exist $\left|\alpha^{\prime}\right|<\left|\alpha_{n}\right|$ such that for $\left|\alpha_{n+1}\right|>\left|\alpha^{\prime}\right|$ we have $P_{d}^{(n+1)}\left(\boldsymbol{\alpha}_{1: n+1}\right)>P_{d}^{(n)}\left(\boldsymbol{\alpha}_{1: n}\right)$ and for $\left|\alpha_{n+1}\right|<\left|\alpha^{\prime}\right|, P_{d}^{(n+1)}\left(\boldsymbol{\alpha}_{1: n+1}\right)<P_{d}^{(n)}\left(\boldsymbol{\alpha}_{1: n}\right)$. This means that for sufficiently large $\left|\alpha_{n+1}\right|$, taking into account the additional path enables us to improve detection performance. While for small $\left|\alpha_{n+1}\right|$, it is better not to account for this path. An asymptotic analytical proof of this conclusion can be found in Appendix B.

This result raises the question of the choice of the optimal number of paths to consider for obtaining the best detection probability.

\section{B. Algorithm for selecting the number of paths}

In order to examine the variation of detection probability with respect to the number of paths, let us examine a simple attenuation model: we assume that $\left|\alpha_{k}\right|=\gamma^{k-1}\left|\alpha_{1}\right|(\gamma<1)$. Thus, the SNR of the k-th path $\left(\left|\alpha_{k}\right|\right)$ is 
smaller than (k-1)-th path $\left(\left|\alpha_{k-1}\right|\right)$ by a certain constant $\mathrm{dB}$ (with respect to a predefined value $\gamma$ ). Fig. 4 illustrates the variation of the detection probability $P_{d}$ with respect to the number of paths taken into account. Different values of $\gamma$ (loss per path) and $\left|\alpha_{1}\right|$ (SNR of the first path) are chosen.

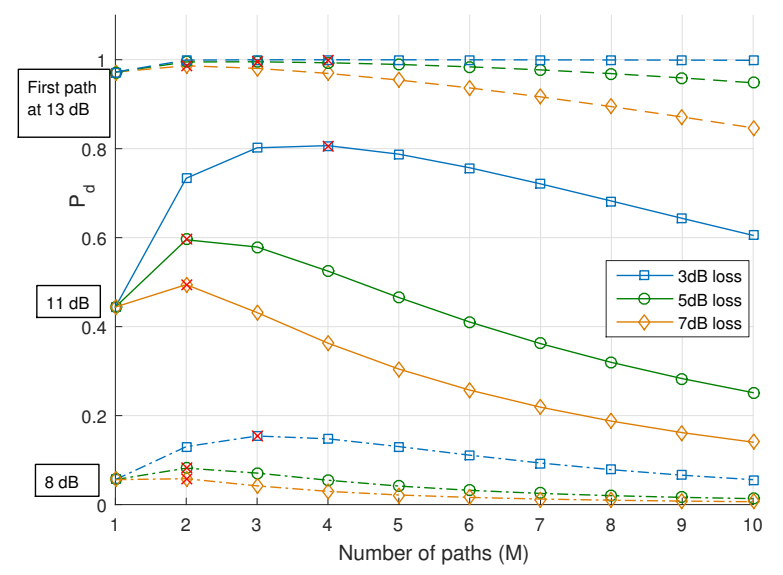

Fig. 4: $P_{d}$ with respect to the number of multiple paths for different SNRs of the first path and different values of the loss factor $\gamma$. Red crosses represent the maximum of $P_{d}$ for each curve.

From this figure, we can observe that for all cases, there indeed exists an optimal value of $\mathrm{M}$ that maximizes $P_{d}^{(M)}$. This can be easily explained: adding one path enables to retrieve more energy from the target but also increases the noise energy gathered; as long as the SNR of the additional path remains strong enough to combat this increase in noise, it will improve the detection performance. This optimal number depends on the SNR of the first path and on the particular loss scheme. Actually, the SNRs of the different paths do not decrease in the simple way described above. In fact, the path loss depends on the geometry of the scene, the reflection coefficients of surrounding surfaces and the target, the position and orientation of the target, etc.

We provide a selection algorithm to select the optimal number of paths for the detection-localization algorithms based on solving the following optimization problem:

$$
M_{o p t}=\underset{M}{\operatorname{argmax}} P_{d}^{(M)}(\boldsymbol{\alpha})
$$

with

$$
P_{d}^{(M)}(\boldsymbol{\alpha})=Q_{M}\left(\sqrt{2\left(\sum_{k=1}^{M}\left|\alpha_{k}\right|^{2}\right)}, \sqrt{2 t_{M}}\right)
$$

where $\left|\alpha_{1}\right| \geq\left|\alpha_{2}\right| \geq \ldots \geq\left|\alpha_{M}\right|, t_{M}=\gamma^{-1}\left(M, \Gamma(M)\left(1-P_{f a}\right)\right)$ and assuming that $\mathbf{r}\left(t-\tau_{i}\right)$ and $\mathbf{r}\left(t-\tau_{j}\right)$ are uncorrelated for all pairs $(i, j)$.

The rationale behind this is that only the paths that positively contribute to the increase of $P_{d}$ should be taken into account.

In real condition, the SNRs of the different paths are not known and have to be estimated from a noisy signal. 


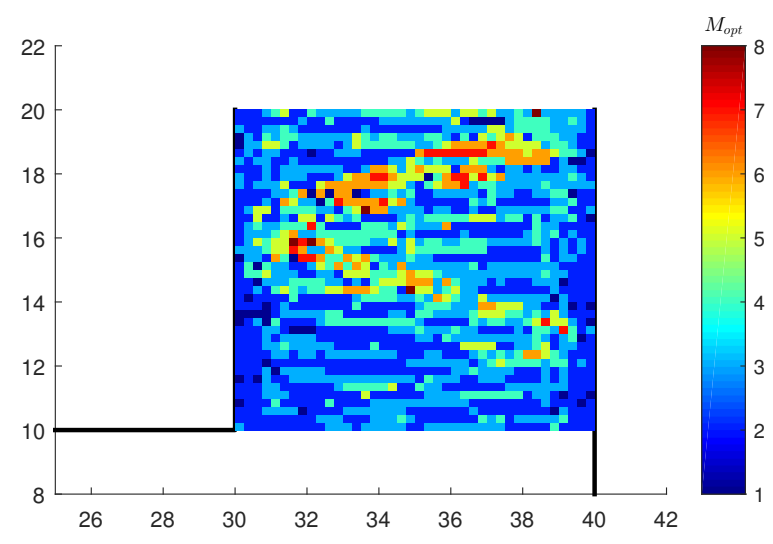

Fig. 5: Map of the optimal number of multiple paths found by the selection algorithm. Simulation carried out with a rough model of an urban intersection described in section II (Fig. 2).

The ML estimation $\hat{\boldsymbol{\alpha}}$ of $\boldsymbol{\alpha}$ given by Equation (4) can be used to estimate $M_{\text {opt }}$ :

$$
\hat{M}_{o p t}=\underset{M}{\operatorname{argmax}} P_{d}^{(M)}(\hat{\boldsymbol{\alpha}}) .
$$

We propose the selection algorithm to choose an optimal number $M$ for maximizing the detection performance: Step 1: Estimate $\hat{\boldsymbol{\alpha}}$ from the measured signal

Step 2: Sort $\left|\hat{\alpha}_{k}\right|$ by decreasing order $\left(\left|\hat{\alpha}_{1}\right| \geq\left|\hat{\alpha}_{2}\right| \geq \ldots \geq\left|\hat{\alpha}_{M}\right|\right)$

Step 3: Calculate $P_{d}^{(M)}\left(\hat{\boldsymbol{\alpha}}_{1: M}\right)$ for different values of $M$

Step 4: Search for the optimal $M\left(\hat{M}_{o p t}\right)$ that maximizes $P_{d}^{(M)}$

The asymptotic convergence of $\hat{M}_{o p t}$ is proved in Appendix B and shows that the algorithm has to stop at some finite value for $M$.

\section{NUMERICAL RESUlts}

In order to evaluate the proposed detection-localization algorithms and the selection algorithm, we carried out a simulation with a rough model of an urban intersection as described in section II (Fig. 2). The area of interest is discretized by $0.25 \mathrm{mx} 0.25 \mathrm{~m}$ cells. In this section, the selection algorithm is first applied to all cells of the area to find the optimal number of paths for each cell. Then the detection performance of different algorithms will be compared by applying them to simulated data obtained from the simulation. Localization performance of the MPF and the SLC on simulated data will also be presented.

\section{A. Selection of the number of paths}

At first, it is desirable to determine the optimal number of paths for all positions in the area of interest in the simulated scenario. By applying the selection algorithm described above, the map of the optimal number for all positions of the target is shown in Fig. 5. We can observe that $M_{\text {opt }}$ has values mostly in the interval from 2 to 4 with the loss scheme considered for the simulation. In some other positions, $M_{o p t}$ is relatively large because there is a high number of strong amplitude path returns due to the scene. 


\section{B. Comparison of detection performance}

In order to evaluate the performance of the MPF and SLC algorithms, let us first define for each path $i$ the propagation loss (including reflection loss) $\nu_{i}$ and the target amplitude $\kappa$ such that $\alpha_{i}=\nu_{i} \kappa$ (we assume here that the target amplitude is identical for all the directions of the paths (no fluctuation of Radar cross-section)). Assuming that the multipath delays are resolved, the signal-to-noise ratio (SNR) is thus

$$
\begin{aligned}
\mathrm{SNR} & =\frac{\|\mathbf{R}(x, y) \boldsymbol{\alpha}\|^{2}}{\sigma^{2}} \\
& \approx \frac{\|\boldsymbol{\alpha}\|^{2}}{\sigma^{2}}=\frac{\left(\sum_{i}\left|\nu_{i}\right|^{2}\right) \kappa^{2}}{\sigma^{2}} .
\end{aligned}
$$

Fig. 6 provides detection performance when the target is located at the reference position $(39.875,10.125)$. Since the loss $\sum_{i}\left|\nu_{i}\right|^{2}$ is constant for a fixed position, the SNR is varied by modifying the value of $\kappa$. The detection probabilities for both MPF and SLC algorithms are calculated using two methods: one with a Monte Carlo simulation and the other with the theoretical formulas (9) and (14). The detection probability of the classic matched filter (MF) for one single path is also computed by Monte Carlo simulation, as well as the detection performance of the clairvoyant matched filter. Its received signal parameters are perfectly known (that is its paths amplitudes and phases are perfectly known, which is unrealistic). At this LOS position, the MPF performance is always better than the MF performance for every SNR. The performance curve of the SLC is very close to that of the MPF one. We can observe a $2 \mathrm{~dB}$ loss between the MPF/SLC and the clairvoyant matched filter. This loss results from parameter estimation with the MPF algorithm or from the incoherent integration with the SLC algorithm.

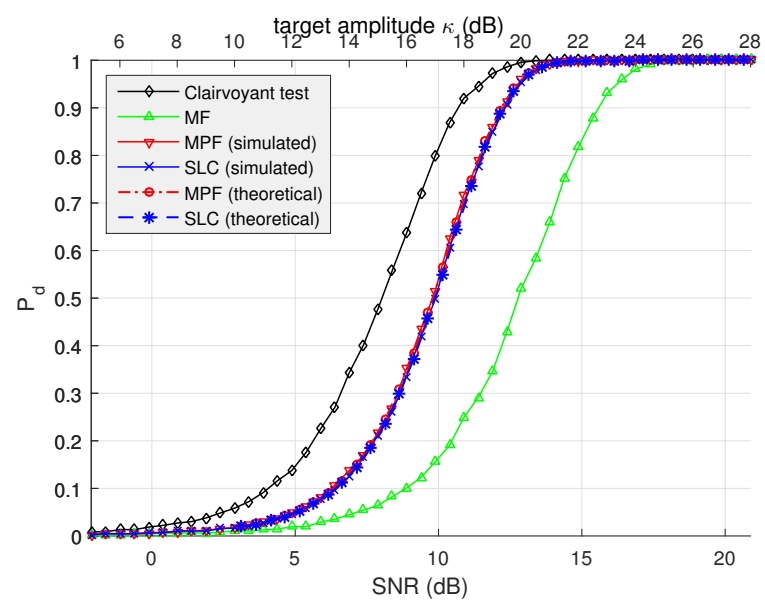

Fig. 6: Detection probability with respect to SNR. Target at the reference position $(39.875,10.125) . M_{o p t}=2$ for MPF and SLC algorithms. $P_{f a}=10^{-3}$. Losses $\sum_{i}\left|\nu_{i}\right|^{2}=-7.12 \mathrm{~dB}$.

In Fig. 7, we present the same curves for an NLOS target located at coordinates $(35.125,12.125)$. It is first noted that all curves are shifted to higher values of $\kappa$, which means that due to propagation loss, a higher level of target amplitude is necessary for detection. We still observe that the MPF and SLC algorithms provide better detection 
performance compared to the MF. However this time we can see a degradation of $1.5 \mathrm{~dB}$ of the SLC compared to the MPF, which is coherent with the theoretical demonstration in section III-C. Depending on the performance considerations, this degradation can be accepted to accelerate the computation time and the memory load.

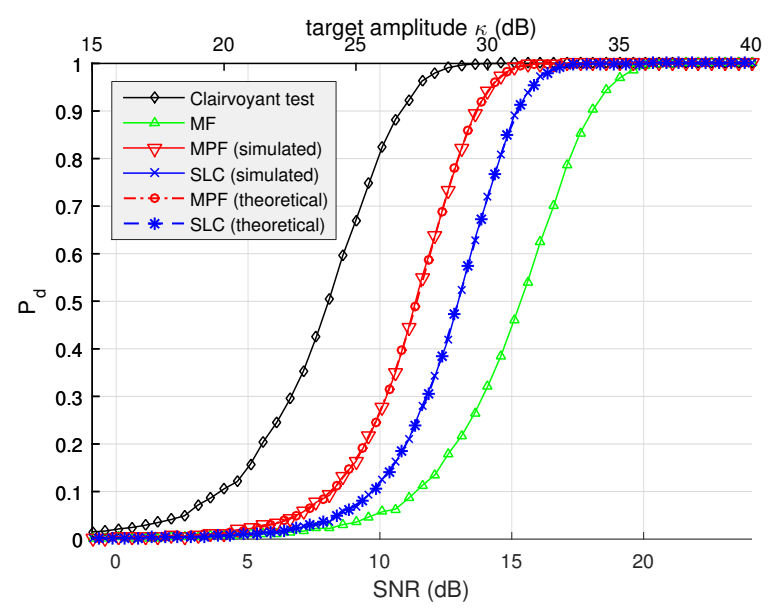

Fig. 7: Detection probability with respect to SNR. Target at coordinates $(35.125,12.125) . M_{o p t}=3$ for MPF and SLC algorithms. $P_{f a}=10^{-3}$. Losses $\sum_{i}\left|\nu_{i}\right|^{2}=-15.91 \mathrm{~dB}$.

\section{Localization results on simulated signals}

We examine two simulations with a target at NLOS positions $(32 ; 14.25)$ and $(37 ; 14.25)$. Results of the MPF localization algorithm are shown in Fig. $8 \mathrm{a}$ and $9 \mathrm{a}$ with respect to the $x$ and $y$ coordinates. Color levels represent the output of the MPF localization algorithm, or T-level. On both maps, we can see that the maximum is close to the true position of the target. Figs.8b and $9 \mathrm{~b}$ present results obtained for the SLC algorithm and also show that the maximum indeed corresponds to the real position of the target.

However, the presence of the target in the scene also created a lot of artifacts, and in many cells the T-level obtained is also relatively high. These high-level cells can be called spatial ambiguities and correspond to the high sidelobes of the proposed spatial multipath matched filter. This is due to the fact that, depending on the scene geometry, there can be several cells whose corresponding multipath delays are quite similar to the delays expected for the true target position. In particular, many different locations may share at least one or two similar delays. These spatial ambiguities are prone to generating local maxima that will not only create ghost false detection, but may also generate a strong bias in the localization performance. These ambiguities, which are due to the geometry of the scene and the imperfect knowledge of the path amplitudes, are very difficult to avoid. We thus observe very similar ambiguities with the MPF and SLC algorithms. This phenomenon is well-known in multipath exploitation [6].

Now let us see how the algorithms perform with a moving target. A target is simulated, moving horizontally from $(30,14.25)$ to $(40,14.25)$ with a uniform speed. The MPF and SLC localization algorithms are applied to 40 equally spaced positions between the two end points. For each position, we thus find the estimated target position 


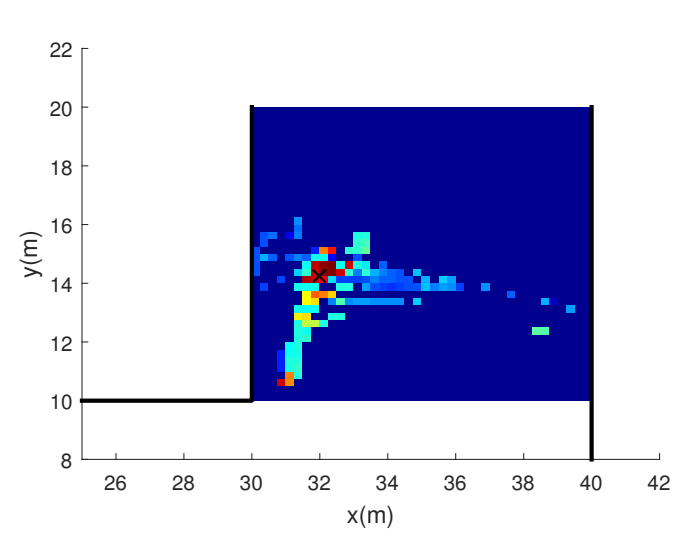

(a) With MPF algorithm
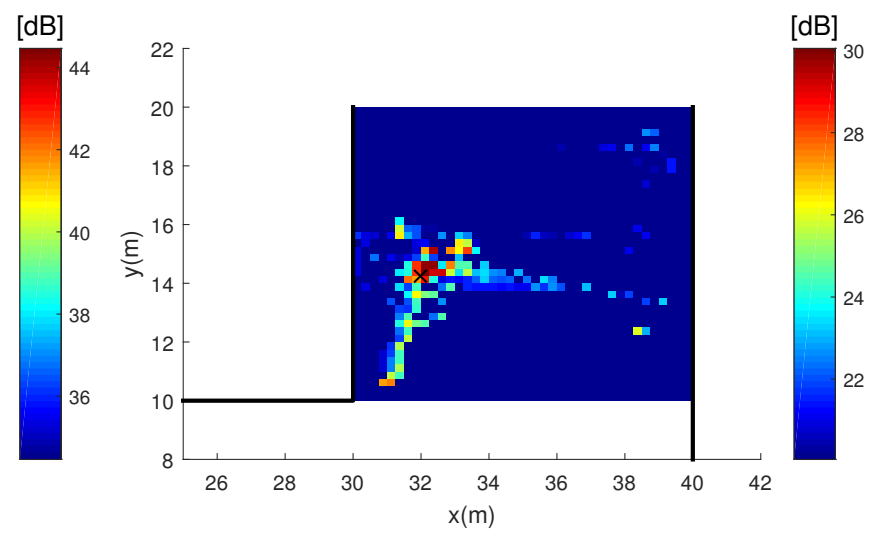

(b) With SLC algorithm.

Fig. 8: Localization result for a target at $(32,14.25)$. The black cross represents the true target position. SNR=15 $\mathrm{dB}$.

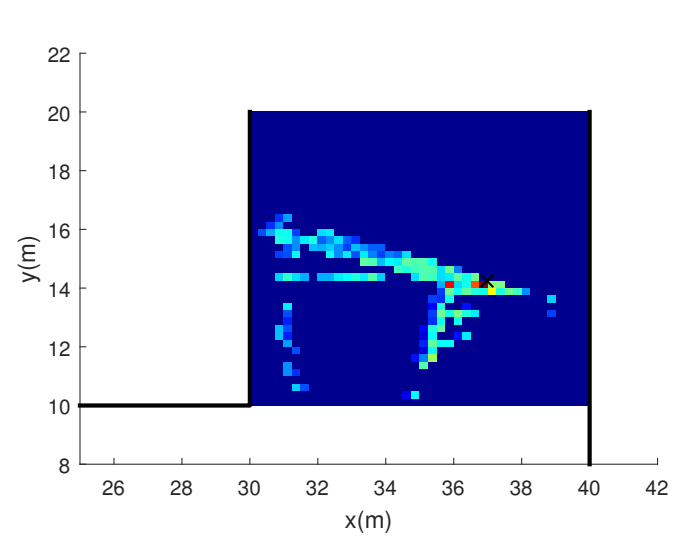

(a) With MPF algorithm
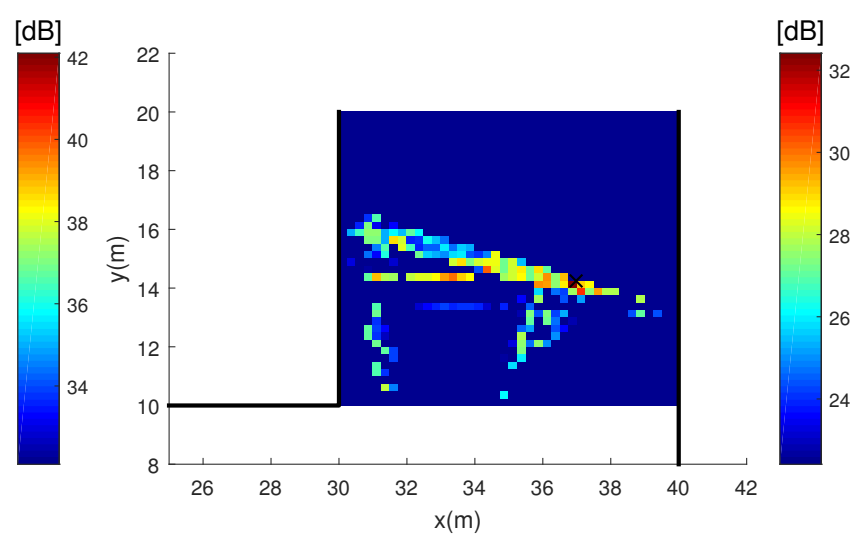

(b) With SLC algorithm.

Fig. 9: Localization result for a target at $(37,14.25)$. The black cross represents the true target position. SNR=15dB.

$(\hat{x}, \hat{y})$ that maximizes the T-level for each algorithm. Then the root mean square error (RMSE) provided by:

$$
\mathrm{RMSE}=\sqrt{\left[\sum_{n=1}^{M C}\left(\left(x-\hat{x}_{n}\right)^{2}+\left(y-\hat{y}_{n}\right)^{2}\right)\right] / M C .}
$$

is calculated by $M C=1000$ Monte Carlo simulations and presented in Fig. 10.

We can observe good agreement between estimated and true positions except at the ends of the trajectory, where multipath information is not sufficient to ensure good localization (i.e. lack of multipath or unresolved multipath). Overall, the RMSE of the MPF algorithm is better than that of the SLC. 


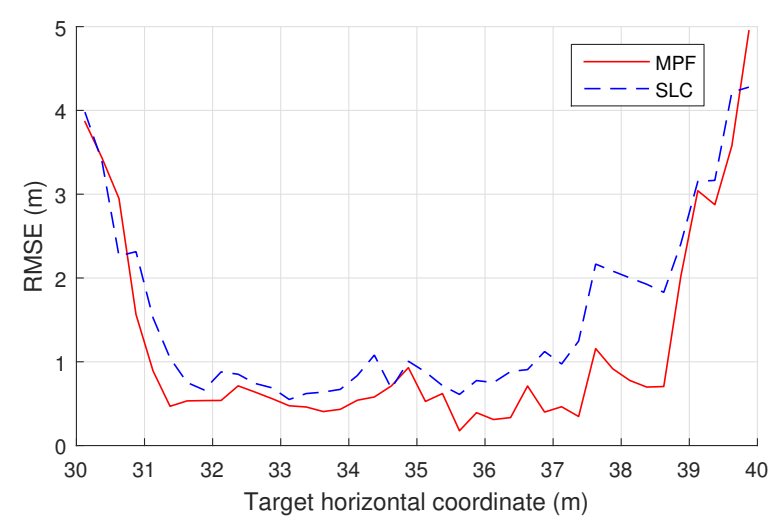

Fig. 10: Localization error along target trajectory. SNR $=15 \mathrm{~dB}$

\section{REAL DATA EXPERIMENT}

\section{A. Experiment description}

In order to verify the practical behavior of the proposed algorithms, an experimentation was set up in a typical urban environment. A T-junction, quite representative of an urban intersection, was selected for the real measurement campaign (Fig. 11). An NLOS target scenario considered in this T-junction is presented in Figure 12. Prior to the experimentation and based on simulations, we chose the target trajectory in order to provide a rich multipath environment.

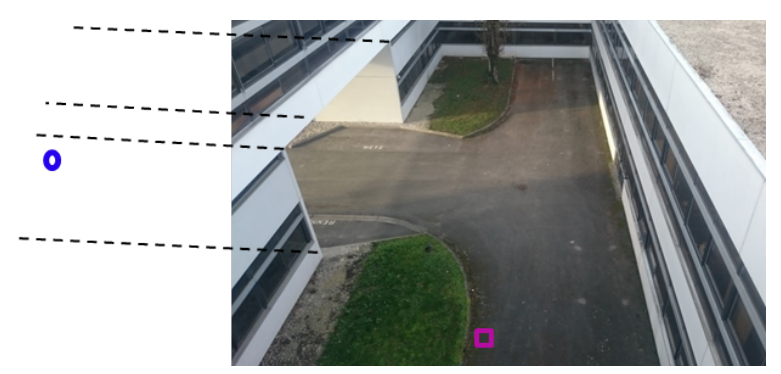

Fig. 11: Photo of the T-junction for the measurement campaign. The radar was placed at the end of the tunnel (blue circle). The target walked toward the right tail of the $\mathrm{T}$ (pink rectangle).

In this scenario, the radar was placed at coordinates $(6.8 ; 0)$ (blue circle), and a pedestrian target moves from $\mathrm{D}(15.5 ; 12.75)$ to $\mathrm{C}(15.5 ; 24.75)$ (pink rectangle) for 15 seconds. An example of the specific multipath propagation for this scenario is shown in Fig. 12. At the beginning of its trajectory, the target was in NLOS position. When the target approached C, it entered the LOS area but multipaths were still also present. This choice of target trajectory enables us to consider and compare two different multipath propagation scenarios: when the target is in NLOS and when the target is in LOS.

For all measurements, we used the $24 \mathrm{GHz}$ 2-Channel FMCW Radar commercialized by IMST. The data were recorded on a laptop via a Raspberry Pi. Note that this radar was not designed for such an application. The signal 


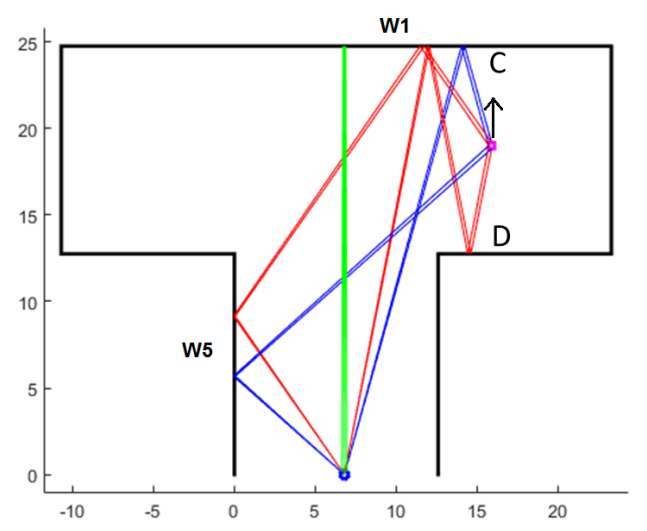

Fig. 12: T-junction scenario considered for the experimentation, in the presence of an NLOS target. Green lines correspond to fixed echoes, blue and red lines represent respectively radar-target paths (or inversely) with singlebounce and double-bounce reflections. W1 and W5 denote two walls involved in the multipath propagation. The scale is in meters.

transmitted by this radar consisted of a coherent train of Linear Frequency Modulation (LFM) pulses. The signal bandwidth was $800 \mathrm{MHz}$, thus corresponding to a range resolution of $0.1875 \mathrm{~m}$. The choice of such a quite large bandwidth was motivated by the need to be able to separate close paths. The duration of each LFM pulse was equal to $T=8 \mathrm{~ms}$, and the pulse repetition period (PRP) was set to $T_{r}=60 \mathrm{~ms}$. This very long PRP, that could not be chosen smaller due to the specific hardware configuration, unfortunately provided a very small Doppler ambiguity (about $0.1 \mathrm{~m} / \mathrm{s}$ ). For such a small Doppler ambiguity, any not perfectly zero-Doppler backscatter is immediately spread over the whole Doppler ambiguity domain and in particular that of the target whose energy is thus diluted. Thus, Doppler processing could not be fully exploited in this setting. Here, it consisted in applying a zero-Doppler rejection [17][18] to remove strong energy at zero Doppler, and then to incoherently integrate the residual energy in all Doppler cells for each range cell. This allows to gather all the energy diluted over the Doppler dimension.

The total duration of each measurement was about $15 \mathrm{~s}$. The radar was placed at $1.2 \mathrm{~m}$ high from the ground. The horizontal aperture of the radar antenna was $60^{\circ}$ and the signal was transmitted with a vertical polarization.

\section{B. Measurement results}

The output of the range matched filter before rejection of the fixed echoes is presented in Fig. 13. It appears that fixed echoes are strong, thus motivating the zero-Doppler rejection procedure explained in the previous section. Measured range profiles after zero-Doppler filtering are presented in Fig. 14. In the NLOS phase, range profiles contain only echoes due to paths reflected at least by one wall. In the LOS phase, the nearest strong echo results from the direct path (without any reflection on surrounding walls). In order to interpret the measurement, a simulation was carried out with a rough geometrical model of the scene. The result is shown in Fig. 15.

Comparing the simulation result in Fig. 15 and the measure in Fig. 14, we can observe that quite good agreement is achieved between simulated echoes generated and the measured echoes. In the NLOS phase, the first three strongest echoes are indicated as 1,2 and 3 in Fig. 14. The echo 1 corresponds to a double-bounce reflection 


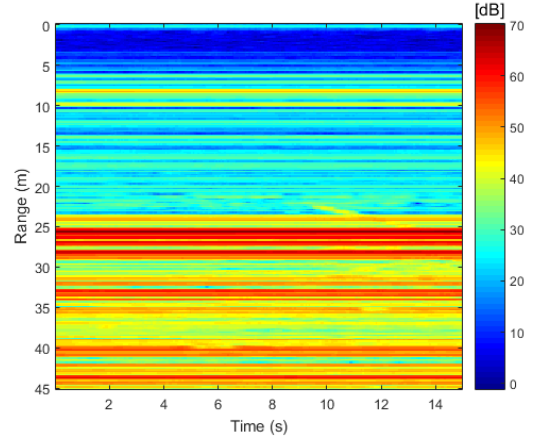

Fig. 13: Range profiles before zero-Doppler filtering

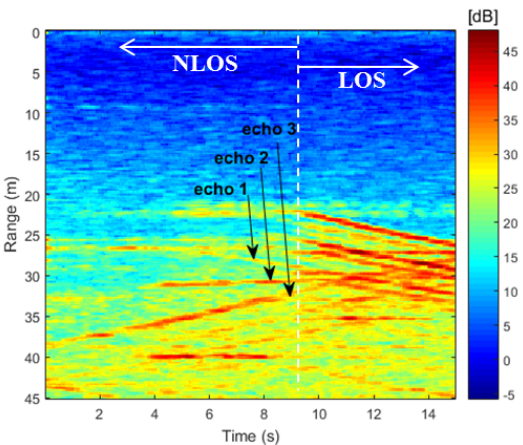

Fig. 14: Range profiles after zeroDoppler filtering

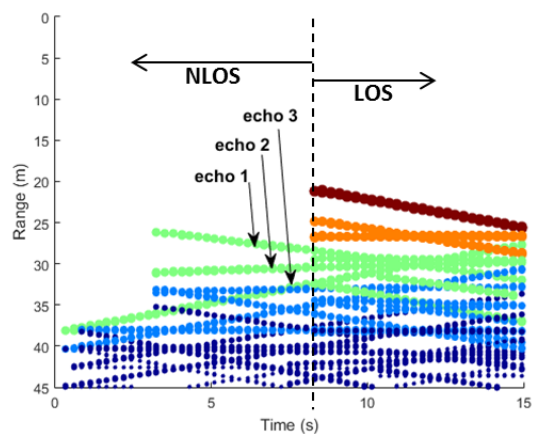

Fig. 15: Simulated range profiles. Red echo: direct return (without wall reflection); orange, green, sky blue and navy blue echoes: returns with one, two, three and four wall reflections respectively.

following the path radar-W5-target-W5-radar. When the target moves away from the radar, the length of this path increases. Conversely, the echo 3 whose length decreases over time corresponds to the path radar-W1-target-W1radar. The echo 2 combines these two paths, i.e. radar-W1-target-W5-radar and conversely. The energy difference can be explained by the fact that the reflection coefficients of the different walls, the loss due to the different scattering angles, and the radar antenna mainlobe were not considered in our propagation model.

Also in this phase, apart from the three apparent echoes, a fuzzy zone can be observed at far ranges. This fuzzy zone is quite well explained by the simulation: as it can be seen in Fig. 15, for range longer than 35m, many path returns can be observed. This also motivates the need to select only a reduced number of paths as we proposed in section IV.

In the LOS phase, the direct return is clearly observed, which is coherent with the red echo in the simulation. The two orange simulated echoes can also be observed. However, some additional echoes can be seen in Fig. 14, which are not explained by the simple propagation result of Fig. 15. These echoes are however less energetic and parallel to the first echo in the LOS phase. The most likely explanation is the simplicity of the geometry used in the simulation: some other possible obstacles in the scene were not modeled in the simulation.

\section{Detection-localization results on measured signals}

MPF and SLC algorithms were applied to experimental data. The algorithms were applied in a specific zone discretized with $0.1875 \mathrm{mx} 0.1875 \mathrm{~m}$ cells. We present in Fig. 16 the result of the MPF localization (a) and SLC localization (b) applied to measured signals when the pedestrian target was located at coordinates (15.5; 16.2). It appears that the maxima of both MPF and SLC test outputs indeed correspond to the true position of the target. This means that, as for localization, the proposed tests provide additional information about the target position by exploiting several paths. Like for localization on simulated scenario described in the section V-C, the presence of the target in the scene creates spatial ambiguities. Fig. 16(c) represents the result of MPF localization for data simulated with a rough geometrical model of the actual scene. We observe a good agreement between the ambiguities in (a), 


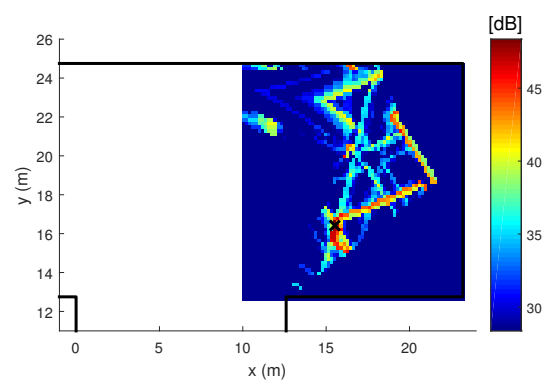

(a) Localization result on measured signal: output of the MPF test

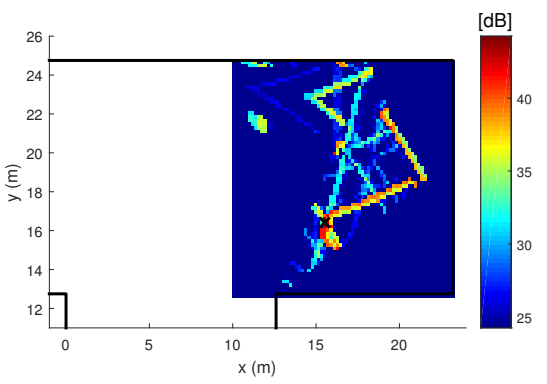

(b) Localization result on measured signal: output of the SLC test

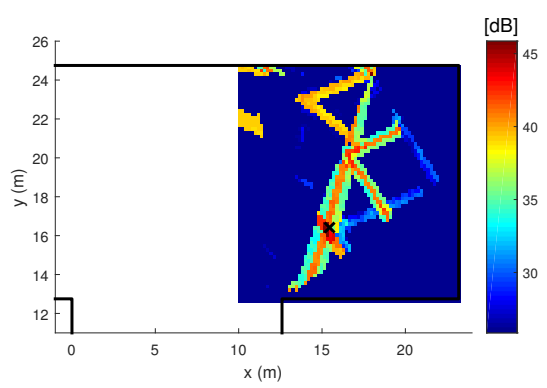

(c) Localization result on simulated signal

Fig. 16: Localization of the target at position $(15.5 ; 16.2)$.

(b) and (c). This means that these ambiguities are due to the geometry of the scene, and thus will be encountered with any method exploiting the geometry.

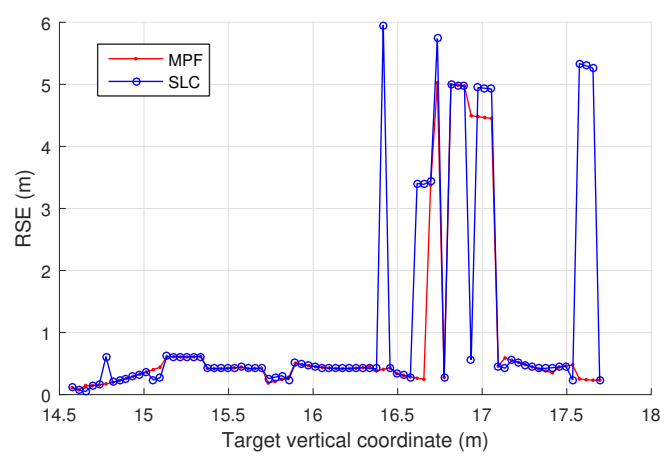

Fig. 17: Position root squared error of the MPF and SLC algorithms when the target moves from $(15.5,14.6)$ to $(15.5,17.7)$

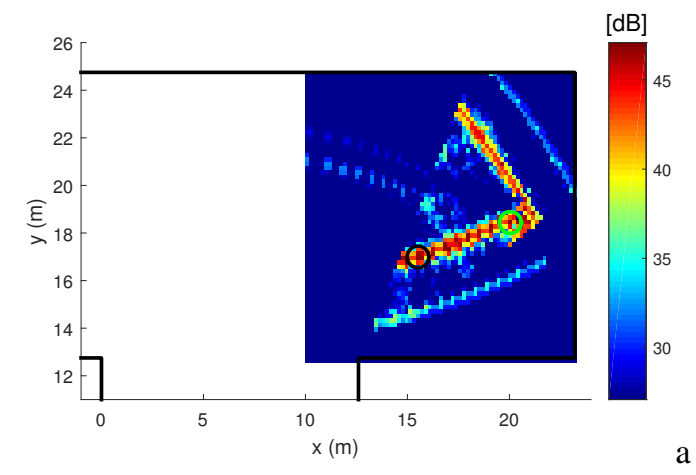

Fig. 18: MPF localization result when the target is at $(15.5,17)$

We now show the localization results for several instances of the target when it is on the NLOS trajectory. In Fig. 17, we present the root square error (RSE) for different target positions. The target location is generally estimated with a high accuracy. With the MPF algorithm, the rate of a localization with MSE $<0.75 \mathrm{~m}$ is $89 \%$. This rate for the SLC algorithm is $82 \%$. This shows that our proposed algorithms using a very simple propagation model (the geometry model considers only wall positions, no information on the permittivity of the reflecting materials nor modeling of fine details such as doors or windows, and a simple ray tracing model for the propagation) provide relevant target position even for real data experiments. Most of the time, the maximum T-level of discretized cells corresponds to the real position of the target. In a few cases though, we observe some false localization, especially when the target y-coordinate is between 16.7 and $17.2 \mathrm{~m}$. This can be explained by high ambiguities resulting from the geometry of the scene. These ambiguities can generate ghosts or strong bias affecting localization performance. For example, Fig. 18 represents the localization result of MPF algorithm for the target coordinates $(15.5,17)$. We can observe a high T-level $(46.56 \mathrm{~dB})$ at the true position of the target (black circle). Unfortunately, due to high 
ambiguities and environmental noise, the algorithm locates the target at a very right shifted position (green circle) that provides a slightly higher T-level $(46.63 \mathrm{~dB})$. Therefore, in that specific case, some post-processing will be necessary to avoid ambiguities. Overall, we should recall that these high ambiguities are inherent to the aroundthe-corner radar localization problem, and that despite of them, the proposed method manages to locate correctly the target most of the time.

\section{CONCLUSION}

In this article, we have presented the problem of around-the-corner radar and proposed two solutions for target detection and localization by exploiting multipath returns. For the detection problem, the proposed algorithms are demonstrated to generally provide better performance than the classic matched filter in a typical NLOS urban scenario. In particular, we show that multipath exploitation can improve detection performance. We have also developed an algorithm for selecting the number of paths so that the detection probability is optimized. For the localization problem, simulated and experimental results show that target locations can be retrieved with both algorithms, even when using a simple propagation model and rough knowledge of the scene geometry. However, it also appears that the localization problem addressed by the "around-the-corner" radar application leads to strong spatial ambiguities which are inherent to the geometry of the scene. Thus, they represent a remaining challenge to be solved. Our ongoing work will be focused on that problem. In future work, the influence of the precision accuracy about the scene (e.g. wall location errors) will be analyzed. We also plan to carry out experiments for more complex scenes.

\section{APPENDIX A}

\section{MPF $P_{F A}$ AND $P_{d}$ DERIVATION}

\section{MPF $P_{F A}$ derivation}

Under hypothesis $H_{0}$ and in the absence of the target, $T_{M P F}^{\prime}(x, y)=T_{M P F}(x, y) / \sigma^{2}$ follows a $\chi^{2}$ central distribution with $2 M(x, y)$ degrees of freedom [21]. The false alarm probability can be computed from the cumulative distribution function $(\mathrm{CDF})$ of the $\chi^{2}$ central distribution as

$$
P_{F A}=1-\frac{\gamma\left(M(x, y), \frac{\lambda_{M P F}^{\prime}}{2}\right)}{\Gamma(M(x, y))},
$$

where $\Gamma(N)$ and $\gamma(N, x)$ are the gamma and the lower incomplete gamma functions respectively. Thus the threshold level of the distribution $T_{M P F}(x, y)$ is given by :

$$
\lambda_{M P F}=\sigma^{2} \lambda_{M P F}^{\prime}=2 \sigma^{2} \gamma^{-1}\left(M(x, y), \Gamma(M(x, y))\left(1-P_{F A}\right)\right) .
$$

\section{MPF $P_{d}$ derivation}

Under hypothesis $H_{1}$ and in the presence of the target, $T_{M P F}^{\prime}(x, y)$ is a $\chi^{2}$ non-central distribution with $2 M(x, y)$ degrees of freedom and the non-centrality parameter $p_{c}=\|\mathbf{R}(x, y) \boldsymbol{\alpha}\|^{2} / \sigma^{2}$ [21]. The detection probability can 
be calculated as

$$
\begin{aligned}
P_{d(M P F)} & =P\left(T_{M P F}(x, y)>\lambda_{M P F}\right) \\
& =P\left(T_{M P F}^{\prime}(x, y)>\lambda_{M P F}^{\prime}\right) \\
& =\int_{\lambda_{M P F}^{\prime}}^{\infty} f_{\chi^{2}}(y) d y,
\end{aligned}
$$

where $f_{\chi^{2}}$ is the probability density function of the $\chi^{2}$ distribution:

$$
\begin{aligned}
f_{\chi^{2}}(y) & =\frac{1}{2} \exp \left(-\left(y+p_{c}\right) / 2\right)\left(\frac{y}{p_{c}}\right)^{(M(x, y)-1) / 2} \\
& \times I_{M(x, y)-1}\left(\sqrt{p_{c} y}\right) .
\end{aligned}
$$

Letting $x=\sqrt{y}$, we have

$$
\begin{aligned}
P_{d(M P F)} & =\int_{\sqrt{\lambda_{M P F}^{\prime}}}^{\infty} \exp \left(-\left(x^{2}+p_{c}\right) / 2\right) \\
& \times x\left(\frac{x^{2}}{p_{c}}\right)^{(M(x, y)-1) / 2} I_{M(x, y)-1}\left(\sqrt{p_{c} x^{2}}\right) d x \\
& =Q_{M(x, y)}\left(\frac{\|\mathbf{R}(x, y) \boldsymbol{\alpha}\|}{\sigma}, \sqrt{\lambda_{M P F}^{\prime}}\right)
\end{aligned}
$$

using the definition of the Marcum Q-function provided in the equation (10)[22].

The derivations of (13) and (14) for SLC $P_{F A}$ and $P_{d}$ are similar.

\section{APPENDIX B}

We will consider two detection probabilities: $P_{d}^{(n)}\left(\boldsymbol{\alpha}_{1: n}\right)$ and $P_{d}^{(n+1)}\left(\boldsymbol{\alpha}_{1: n+1}\right)$ when $n$ and $n+1$ paths are taken into account respectively. We assume that all paths are relatively separated, so that $\mathbf{r}\left(t-\tau_{i}\right)$ and $\mathbf{r}\left(t-\tau_{j}\right)$ are uncorrelated for any pairs of $(i, j)$ and without of generality that $\left|\alpha_{1}\right| \geq\left|\alpha_{2}\right| \geq \ldots \geq\left|\alpha_{n+1}\right|$. Thus, the detection probabilities of the MPF with $n$ and $n+1$ multipaths are respectively provided by:

$$
\begin{aligned}
P_{d}^{(n)}\left(\boldsymbol{\alpha}_{1: n}\right) & =Q_{n}\left(\sqrt{2 \sum_{k=1}^{n}\left|\alpha_{k}\right|^{2}}, \sqrt{2 t_{n}}\right) \\
& =Q_{n}\left(\sqrt{2 \beta^{2}}, \sqrt{2 t_{n}}\right)
\end{aligned}
$$

and

$$
\begin{aligned}
P_{d}^{(n+1)}\left(\boldsymbol{\alpha}_{1: n+1}\right) & =Q_{n+1}\left(\sqrt{2 \sum_{k=1}^{n+1}\left|\alpha_{k}\right|^{2}}, \sqrt{2 t_{n+1}}\right) \\
& =Q_{n+1}\left(\sqrt{2\left(\beta^{2}+\left|\alpha_{n+1}\right|^{2}\right)}, \sqrt{2 t_{n+1}}\right),
\end{aligned}
$$


where $\beta^{2}=\sum_{k=1}^{n}\left|\alpha_{k}\right|^{2}, t_{n}=\gamma^{-1}\left(n, \Gamma(n)\left(1-P_{f a}\right)\right), t_{n+1}=\gamma^{-1}\left(n+1, \Gamma(n+1)\left(1-P_{f a}\right)\right.$ and the Marcum Q-function is defined as:

$$
Q_{M}(a, b)=\int_{b}^{\infty} x\left(\frac{x}{a}\right)^{M-1} \exp \left(-\frac{x^{2}+a^{2}}{2}\right) I_{M-1}(a x) d x
$$

Let us note

$$
\Delta P_{n+1}\left(\beta,\left|\alpha_{n+1}\right|\right)=P_{d}^{(n+1)}\left(\boldsymbol{\alpha}_{1: n+1}\right)-P_{d}^{(n)}\left(\boldsymbol{\alpha}_{1: n}\right)
$$

it comes that

$$
\begin{aligned}
\Delta P_{n+1}\left(\beta,\left|\alpha_{n+1}\right|\right) & =Q_{n+1}\left(\sqrt{2\left(\beta^{2}+\left|\alpha_{n+1}\right|^{2}\right)}, \sqrt{2 t_{n+1}}\right) \\
& -Q_{n}\left(\sqrt{2 \beta^{2}}, \sqrt{2 t_{n}}\right) .
\end{aligned}
$$

Numerical simulation shows that it exists $\left|\alpha^{\prime}\right| \in\left(0,\left|\alpha_{n}\right|\right)$ so that $\Delta P_{n+1}\left(\beta,\left|\alpha_{n+1}\right|\right)>0$ when $\left|\alpha_{n+1}\right|>\left|\alpha^{\prime}\right|$ and $\Delta P_{n+1}\left(\beta,\left|\alpha_{n+1}\right|\right)<0$ when $\left|\alpha_{n+1}\right|<\left|\alpha^{\prime}\right|$. It means that the selection algorithm described in the section IV has to stop sooner or later when the amplitudes of longer paths are so small (due to propagation losses). In an asymptotic way, we will show that:

- (*): $\Delta P_{n+1}\left(\beta,\left|\alpha_{n+1}\right|\right)$ increases with $\left|\alpha_{n+1}\right|$

- (**): $\Delta P_{n+1}(\beta, 0)<0$ and $\Delta P_{n+1}\left(\beta,\left|\alpha_{n+1}\right|=\left|\alpha_{n}\right|\right)>0$, for the hypothesis that $\beta$ is sufficiently large.

To prove $(*)$, let us remark that

$$
\frac{\partial \Delta P_{n+1}\left(\beta,\left|\alpha_{n+1}\right|\right)}{\partial\left|\alpha_{n+1}\right|}=\frac{\partial Q_{n+1}\left(\sqrt{2\left(\beta^{2}+\left|\alpha_{n+1}\right|^{2}\right)}, \sqrt{2 t_{n+1}}\right)}{\partial\left|\alpha_{n+1}\right|}
$$

and that

$$
\frac{\partial Q_{n+1}\left(\sqrt{2\left(\beta^{2}+\left|\alpha_{n+1}\right|^{2}\right)}, \sqrt{2 t_{n+1}}\right)}{\partial\left|\alpha_{n+1}\right|}>0,
$$

since $Q_{M}(a, b)$ increases with $a$ [24]. Thus

$$
\frac{\partial \Delta P_{n+1}\left(\beta,\left|\alpha_{n+1}\right|\right)}{\partial\left|\alpha_{n+1}\right|}>0
$$

Therefore, $\Delta P_{n+1}\left(\beta,\left|\alpha_{n+1}\right|\right)$ increases as $\left|\alpha_{n+1}\right|$ increases in the interval $\left[0 ;\left|\alpha_{n}\right|\right]$. (*) is proved!

To prove $(* *)$, we can write that $\Delta P_{n+1}(\beta, 0)=Q_{n+1}\left(\beta \sqrt{2}, \sqrt{2 t_{n+1}}\right)-Q_{n}\left(\beta \sqrt{2}, \sqrt{2 t_{n}}\right)$.

From [25], [26] and [27], we have:

$$
\begin{aligned}
\frac{\partial Q_{M}(a, b)}{\partial a} & =a\left[Q_{M+1}(a, b)-Q_{M}(a, b)\right] \\
& =a\left(\frac{b}{a}\right)^{M} \exp \left(-\frac{a^{2}+b^{2}}{2}\right) I_{M}(a b) .
\end{aligned}
$$


Then, let

$$
\begin{aligned}
f_{n+1}(\beta) & =\frac{\partial \Delta P_{n+1}(\beta, 0)}{\partial \beta} \\
& =\beta^{-n+1} \sqrt{2} t_{n+1}^{(n+1) / 2} \exp \left[-\left(\beta^{2}+t_{n+1}\right)\right] I_{n}\left(2 \beta \sqrt{t_{n}}\right) \\
& \times\left[\frac{1}{\beta} \frac{I_{n+1}\left(2 \beta \sqrt{t_{n+1}}\right)}{I_{n}\left(2 \beta \sqrt{t_{n}}\right)}-\frac{t_{n}^{n / 2}}{t_{n+1}^{(n+1) / 2}} \exp \left(t_{n+1}-t_{n}\right)\right]
\end{aligned}
$$

We have $\beta^{-n+1} \sqrt{2} t_{n+1}^{(n+1) / 2} \exp \left[-\left(\beta^{2}+t_{n+1}\right)\right] I_{n}\left(2 \beta \sqrt{t_{n}}\right)>0$ and from [28]:

$$
\begin{aligned}
\lim _{\beta \rightarrow+\infty} \frac{1}{\beta} \frac{I_{n+1}\left(2 \beta \sqrt{t_{n+1}}\right)}{I_{n}\left(2 \beta \sqrt{t_{n}}\right)} & =\lim _{\beta \rightarrow+\infty} \frac{1}{\beta} \sqrt[4]{\frac{t_{n}}{t_{n+1}}} \frac{\exp \left(2 \beta \sqrt{t_{n+1}}\right)}{\exp \left(2 \beta \sqrt{t_{n}}\right)} \\
& =+\infty
\end{aligned}
$$

(as $t_{n+1}>t_{n}$ )

In addition, $\frac{t_{n}^{(n) / 2}}{t_{n+1}^{(n+1) / 2}} \exp \left(t_{n+1}-t_{n}\right)$ is a positive constant. Therefore,

$$
\lim _{\beta \rightarrow+\infty} f_{n+1}(\beta)=+\infty .
$$

As $f_{n+1}(\beta)$ is continuous for $\beta \in(0,+\infty)$; so there exists $\beta_{1}$ so that for any $\beta>\beta_{1}, f_{n+1}(\beta)$ is positive. That is, $\Delta P_{n+1}(\beta, 0)$ increases in the interval $\left[\beta_{1},+\infty\right]$. In addition: $\Delta P_{n+1}(\beta, 0)$ is continuous and $\lim _{\beta \rightarrow+\infty} \Delta P_{n+1}(\beta, 0)=1-1=0$. Therefore $\Delta P_{n+1}(\beta, 0)<0$ for any $\beta>\beta_{1}$.

In the same way, we can show that there exists $\beta_{2}$ so that $\Delta P_{n+1}\left(\beta,\left|\alpha_{n+1}\right|=\left|\alpha_{n}\right|\right)>0$ for any $\beta>\beta_{2}$.

So, letting $\beta_{0}=\max \left(\beta_{1}, \beta_{2}\right)$, it comes that for any $\beta>\beta_{0}: \Delta P_{n+1}(\beta, 0)<0$ and $\Delta P_{n+1}\left(\beta,\left|\alpha_{n+1}\right|=\left|\alpha_{n}\right|\right)>$ $0 .(* *)$ is proved!

\section{REFERENCES}

[1] A. Sume, M. Gustafsson, M. Herberthson, A. Jänis, S. Nilsson, J. Rahm, and A. Örbom, "Radar Detection of Moving Targets Behind Corners," IEEE Transactions on GeoScience and Remote Sensing, vol. 49, pp. 2259-2267, 2011.

[2] O. Rabaste, E. Colin-Koeniguer, D. Poullin, A. Cheraly, J.-F. Pétex, and H.-K. Phan, "Around-the-Corner Radar : Detection of a Human Being in Non Line of Sight," IET Radar, Sonar and Navigation, vol. 9, no. 6, pp. 660-668, 2015.

[3] D. Tahmoush, J. Silvious, and B. Bender, "Radar surveillance in urban environments," in Proceedings of the IEEE International Radar Conference, 2012, pp. 220-225.

[4] B. Chakraborty, Y. Li, J. J. Zhang, and T. Trueblood, "Multipath exploitation with adaptive waveform design for tracking in urban terrain," in Proceedings of the IEEE International Conference on Acoustics, Speech and Signal Processing, 2010, pp. 3894-3897.

[5] R. Linnehan and J. Schindler, "Multistatic scattering from moving targets in multipath environments," in Proceedings of the IEEE International Radar Conference, 2009, pp. 1-6.

[6] P. Setlur, T. Negishi, N. Devroye, and D. Erricolo, "Multipath exploitation in non-LOS urban synthetic aperture radar," IEEE Journal of Selected Topics in Signal Processing, vol. 8, no. 1, pp. 137-152, 2014.

[7] R. Zetik, M. Eschrich, S. Jovanoska, and R. Thoma, "Looking behind a corner using multipath-exploiting UWB radar," IEEE Trans. on Aerospace and Electronic Systems, vol. 51, no. 3, pp. 1916-1926, 2015. 
[8] M. Gustafsson, A. Andersson, T. Johansson, S. Nilsson, and A. Sume, "Extraction of Human Micro-Doppler Signature in an Urban Environment Using a "Sensing-Behind-the-Corner" Radar," IEEE Transactions on GeoScience and Remote Sensing, vol. 13, pp. 187-191, 2016.

[9] T. Johansson, A. Örbom, A. Sume, J. Rahm, S. Nilsson, M. Herberthson, M. Gustafsson, and A. Andersson, "Radar measurements of moving objects around corners in a realistic scene," in Proceedings of SPIE, Radar Sensor Technology, vol. 9077, 2014.

[10] T. Johansson, A. Andersson, M. Gustafsson, and S. Nilsson, "Positioning of moving non-line-of-sight targets behind a corner," in Proceedings of the 13th European Radar Conference, 2017.

[11] P. Setlur, G. Smith, F. Ahmad, and M. Amin, "Target localization with a single sensor via multipath exploitation," IEEE Transactions on Aerospace and Electronic Systems, vol. 48, no. 3, pp. 1996-2014, 2012.

[12] P. Setlur and N. Devroye, "Multipath exploited bayesian and cramer-rao bounds for single-sensor target localization," EURASIP Journal on Advances in Signal Processing, vol. 2013, no. 1, p. 53, 2013.

[13] A. O'connor, P. Setlur, and N. Devroye, "Single-sensor RF emitter localization based on multipath exploitation," IEEE Transactions on Aerospace and Electronic Systems, vol. 51, no. 3, pp. 1635-1651, 2015.

[14] P. Barbosa, E. Chong, S. Suvorova, and B. Moran, "Multitarget multisensor tracking in an urban environment: A closed-loop approach," in Proceedings of the Conference on Signal and Data Processing of Small Targets 2008, part of the SPIE Symposium on Defense and Security, vol. 6969, 2008, pp. 69690W1-69690W12.

[15] A. Weiss, "Direct geolocation of wideband emitters based on delay and Doppler," IEEE Transactions on Signal Processing, vol. 59, no. 6, pp. 2513-2521, 2011.

[16] K. Thai, O. Rabaste, J. Bosse, D. Poullin, I. Hinostroza, T. Letertre, and T. Chonavel, "Around-the-corner radar: Detection and localization of a target in non-line of sight," in Radar Conference (RadarConf), 2017 IEEE, 2017.

[17] F. Colone, D. W. O. Hagan, P. Lombardo, and C. J. Baker, "A multistage processing algorithm for disturbance removal and target detection in passive bistatic radar," IEEE Transactions on Aerospace and Electronic Systems, vol. 45, no. 2, pp. 698-722, apr 2009.

[18] J. Bosse, O. Rabaste, and D. Poullin, "Matching pursuit via continuous resolution cell rejection in presence of unresolved radar targets," in Proceedings of the European Signal Processing Conference (EUSIPCO), 2015, pp. 1816-1820.

[19] M. A. Richards, Fundamentals of Radar Signal Processing. Tata McGraw-Hill Education, 2005.

[20] H. V. Poor, An introduction to signal detection and estimation. Springer Science \& Business Media, 2013.

[21] L. Scharf and B. Friedlander, "Matched Subspace Detectors," IEEE Transactions on Signal Processing, vol. 42, no. 8, pp. 2146-2157, 1994.

[22] D. Shnidman, "The calculation of the probability of detection and the generalized Marcum Q-function," IEEE Transactions on Information Theory, 1989.

[23] J. Proakis and M. Salehi, Communication Systems Engineeing. Prentice Hall, 2002, ch. 10, pp. 689-694.

[24] A. Gil, J. Segura, and N. M. Temme, "The asymptotic and numerical inversion of the Marcum Q-function," Studies in Applied Mathematics, 2014.

[25] A. H. Nuttall, "Some integrals involving the Qm function," IEEE Transactions on Information Theory, vol. 21, pp. 95-96, 1975.

[26] R. Esposito, "Comment on partial differentials of Marcum's Q-function," in Proceedings of the IEEE, vol. 56, Dec. 1968, p. 2195.

[27] Y. A. Brychkov, “On some properties of the Marcum's Q function,” Integral Transforms and Special Functions, pp. 177-182, 2012.

[28] A. AlSaammare, M. Shaqfeh et al., "A simple and efficient approximation to the modified bessel functions and its applications to rician fading," IEEE GCC Conference and Exhibition (GCC), 2013. 


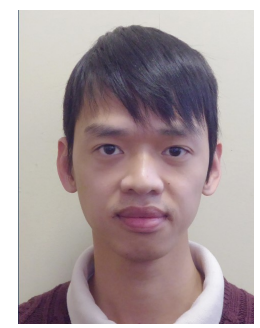

Khac-Phuc-Hung THAI received the Engineering degree from INSA Centre Val de Loire, Blois, France, in 2015 and the PhD degree in Signal, Image and Vision from IMT Atlantique, Brest, France, in 2018. From 2015 to 2018 he worked towards the Ph.D. degree at ONERA, The French Aerospace Lab, Palaiseau, France, where his research interests concern detection and localization in around-the-corner radar.

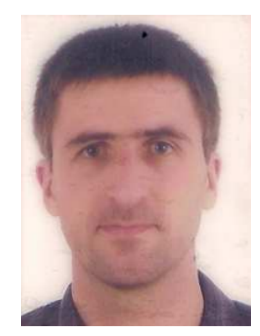

Olivier Rabaste received the Engineering degree in telecommunications in 2003 from the Ecole Nationale Supérieure des Télécommunications de Bretagne, Brest, France, the M.S. degree in digital communications systems and technologies with distinction in 2003 from Chalmers University of Technology, Göteborg, Sweden, and the Ph.D. degree in 2006 from the Ecole Nationale Supérieure des Télécommunications de Bretagne. He is currently a Research Engineer at ONERA, the French Aerospace Laboratory, Palaiseau Cedex, France, since 2008. His research interests include detection, estimation, and tracking for radar applications.

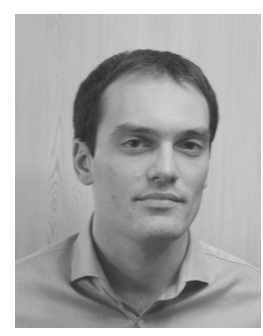

Jonathan Bosse received the M.Sc. degree from Centrale Marseille, France, in 2008 and the PhD degree in Electrical Engineering from Ecole Normale Supérieure de Cachan, France, in 2012. From 2009 to 2012 he worked towards the Ph.D. degree through a CIFRE convention between Thales Communications and Ecole Normale Supérieure de Cachan. He was then Post-doctorate at TU Delft, The Netherlands in the Microwave Sensing, Signals and Systems group during two years. In 2014, he joined ONERA, The French Aerospace Lab, where his research interests still concern estimation and detection in radar and array processing.

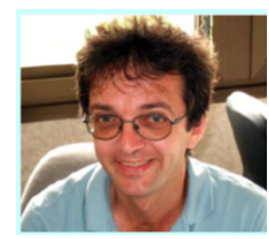

Dominique Poullin is graduated from ENSTA (Ecole Nationale Supérieure des Techniques Avancées) a French High School. He joined ONERA (Office National d'Etudes et de Recherches Aérospatiale) in 1989 where he works especially in the passive radar field. He began with the analog TV waveform, and since 1995's he studied the digital COFDM waveform (Coded Orthogonal Frequency Division Multiplex) and its properties against multipaths and/or multiple transmitters in SFN (Single Frequency Network) mode. He is the author of two patents dealing with the clutter (and direct path) cancellation in passive radar using COFDM waveform. After this focus on the DAB (Digital Audio Broadcasting) standard, he came back to the analog transmitters with FM and he is currently working on DVB-T. He is responsible of the different passive radar studies in ONERA among which: passive DVB-T capabilities against UAV, MIMO-FM (or a way of evaluating target elevation according to the transmitting pylon), passive airborne DVB-T evaluation,... He has participated in several NATO groups on passive radar and he serves as voluntary reviewer for a number of international technical journals. 


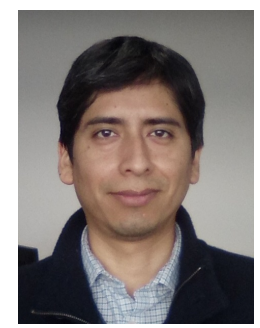

Israel D. Hinostroza Sáenz (M'12) received the B.Sc. degree in electronics engineering from the Universidad Nacional de Ingenieria, Lima, Peru in 2003, the engineering degree and the M.S. degree in microelectronics and telecommunications from the Ecole Polytechnique Universitaire de Marseille, France, in 2009, and the Ph.D. degree in electromagnetics from the Ecole Superieure d'Electricite (Supelec, 2013) from the Supelec ONERA NUS DSO Research Alliance (SONDRA), Gif-Sur-Yvette, France. Since 2013, he has been an Assistant Professor at SONDRA, CentraleSupelec, where he is currently working on antenna arrays and radar applications.

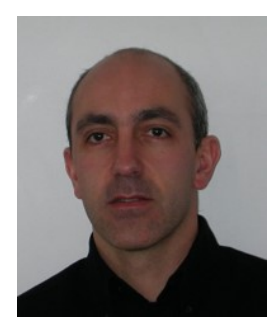

Thierry Letertre obtained is D.E.S.S of Industrial engineering, option Electronic Systems from the University of Paris XI in 1992, the Specialized Degree in Computer Systems and Networks from the "Ecole Supérieure d'Electricité" (CentraleSupélec) in 1998 and received the $\mathrm{PhD}$ degree in Science speciality: Electromagnetism from CentraleSupélec in 2013. He work in Sondra (Supelec ONERA NUS DSO Research Alliance) laboratory and is professor at CentraleSupélec in the department of Telecommunications (since 1998). He is spcialized in Electromagnetics measuremts (EM), EM compatibility, exposure of people to EM fields and is currently working on EM waves propagation modeling and radar applications.

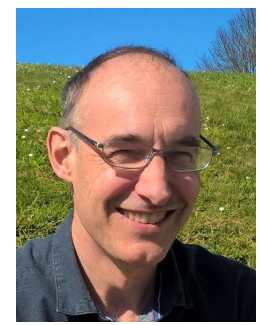

Thierry Chonavel received the Master degree from IMT Atlantique (Télécom Bretagne) in 1987. After working for Alcatel, he joined Télécom ParisTech where he obtained the Ph.D. degree in signal and image processing in 1992. Since then, it has been working for IMT Atlantique has an associate professor and then a professor (since 2004). His researches are related to statistical signal processing, with applications in several areas such as transmissions, radar, sonar or array processing. 


\section{LIST OF FIGURES}

1 Urban radar looking for targets in shadow areas . . . . . . . . . . . . . . . . .

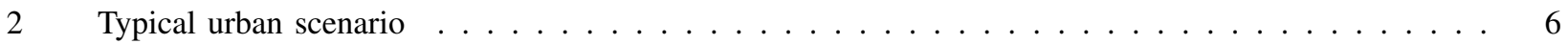

$3 \quad P_{d}^{(n+1)}\left(\boldsymbol{\alpha}_{1: n+1}\right)-P_{d}^{(n)}\left(\boldsymbol{\alpha}_{1: n}\right)$ for different values of $n \ldots \ldots$

$4 \quad P_{d}$ with respect to the number of multiple paths for different SNRs of the first path and different values of the loss factor $\gamma$. Red crosses represent the maximum of $P_{d}$ for each curve. . . . . . . . . . . . 11

5 Map of the optimal number of multiple paths found by the selection algorithm. Simulation carried out with a rough model of an urban intersection described in section II (Fig. 2). . . . . . . . . . . . . . . 12

6 Detection probability with respect to SNR. Target at the reference position $(39.875,10.125) . M_{o p t}=2$

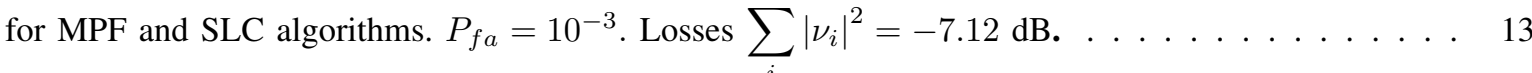

7 Detection probability with respect to SNR. Target at coordinates $(35.125,12.125) . M_{o p t}=3$ for MPF and SLC algorithms. $P_{f a}=10^{-3}$. Losses $\sum\left|\nu_{i}\right|^{2}=-15.91 \mathrm{~dB} \ldots \ldots \ldots$. . . . . . . . . . 14

8 Localization result for a target at $(32,14.25)$. The black cross represents the true target position. $\mathrm{SNR}=15 \mathrm{~dB} \ldots \ldots \ldots \ldots \ldots \ldots \ldots \ldots$

9 Localization result for a target at (37,14.25). The black cross represents the true target position. $\mathrm{SNR}=15 \mathrm{~dB} . \ldots \ldots \ldots \ldots \ldots \ldots \ldots$

10 Localization error along target trajectory. $\mathrm{SNR}=15 \mathrm{~dB} \ldots \ldots \ldots$

11 Photo of the T-junction for the measurement campaign. The radar was placed at the end of the tunnel (blue circle). The target walked toward the right tail of the $\mathrm{T}$ (pink rectangle). . . . . . . . . . 16

12 T-junction scenario considered for the experimentation, in the presence of an NLOS target. Green lines correspond to fixed echoes, blue and red lines represent respectively radar-target paths (or inversely) with single-bounce and double-bounce reflections. W1 and W5 denote two walls involved in the multipath propagation. The scale is in meters. . . . . . . . . . . . . . . . . . . 17

13 Range profiles before zero-Doppler filtering . . . . . . . . . . . . . . . . 18

14 Range profiles after zero-Doppler filtering . . . . . . . . . . . . . . . . . . 18

15 Simulated range profiles. Red echo: direct return (without wall reflection); orange, green, sky blue and navy blue echoes: returns with one, two, three and four wall reflections respectively. . . . . . . . . 18

16 Localization of the target at position $(15.5 ; 16.2) \ldots \ldots \ldots$

17 Position root squared error of the MPF and SLC algorithms when the target moves from $(15.5,14.6)$ to $(15.5,17.7) \ldots \ldots \ldots \ldots \ldots \ldots \ldots$

18 MPF localization result when the target is at $(15.5,17) \ldots \ldots \ldots$ 